\title{
The Effects of Maitland Orthopedic Manual Physical Therapy on the Digestive System
}

\begin{abstract}
The purpose of the present study is to apply Maitland orthopedic manual physical therapy to patients to examine the effects of the therapy on the digestive system through serum gastrin tests that can identify the motility of the digestive system and dyspepsia symptoms and can provide basic data for internal medicinal physical therapy. Maitland orthopedic manual physical therapy was implemented on 20 subjects in total, with 10 in a dyspepsia group and 10 in a control group, for 20 minutes per day, three days per week for three weeks, and the following results were obtained.

In the control group, serum gastrin values significantly decreased between before and after treatment(p<.01), and among questionnaire items regarding dyspepsia, those regarding epigastric pain and belching showed significant decreases in these symptoms(p<.05). In the dyspepsia group, serum gastrin values significantly decreased between before and after treatment( $p(.01)$ and all questions regarding dyspepsia showed significant decreases in all symptoms(p<.01). According to the analysis, the dyspepsia group decreased significantly more than the control group in serum gastrin values and all dyspepsia symptoms, except for belching(p<.001).

Through the present study, it was identified that Maitland orthopedic manual physical therapy can improve dyspepsia symptoms and is an effective treatment method for internal diseases, such as dyspepsia, by improving gastric motility to become close to the normal state.
\end{abstract}

Key words: Dyspepsia; Gastrin; Maitland orthopedic manual physical therapy

\author{
Ja Pung Koo ${ }^{a}$, Nyeon Jun Kim \\ aPohang College, Pohang, Korea \\ Received : 28 October 2014 \\ Revised : 2 December 2014 \\ Accepted : 30 February 2015 \\ Address for correspondence \\ Nyeon Jun Kim, PT, Ph.D \\ Department of Physical Therapy, \\ Pohang College, 55, Sinduk-ro, \\ Heunghae-eup, Pohang, Korea \\ Tel: 82-54-245-1217 \\ E-mail: knj@pohang.ac.kr
}

\section{INTRODUCTION}

Symptoms of digestive system diseases are very common in the general population(1). Researchers reported that some patients who visited digestive clinics complained of dyspepsia with persisting symptoms, such as nausea, vomiting, and belching, without any organic abnormality $(2,3)$. Dyspepsia medically refers to continuous or repetitive epigastric discomfort or epigastric pain limited to the precordium and it is also explained as all symptoms assumed to have occurred in the stomach or the duodenum(4). Dyspepsia is important because it shows high prevalence rates of approximately 30 40\% in sample population surveys and it is known to occur newly in at least approximately $1 \%$ of the population every year. Therefore, it has been reported to have very large socioeconomic effects(5). Dyspepsia is a very common disease with prevalence rates of $20 \sim 30 \%$ globally $(6,7)$.

It has been reported that excessive secretion of gastric acid, delays in digestive system movements, chronic gastritis, infection with Helicobacter pylori, duodenitis, mental factors, such as emotions and stress, and genetic factors are involved in the pathological physiology of dyspepsia(8). Among many causes of the disease reported thus far, the decline of the gastric emptying function due to the movement dysfunction of the digestive system is regarded to be the most important, and the degree of the causal relationship between dyspepsia and the gastric emptying function has been reported to be approximately 30 80\%(9). 
It has been told that many elements are necessary to improve the motility of the digestive system and among them, the actions of digestive system hormones are the most closely involved in the motility of the digestive system(10). Based on information given thus far by studies indicating that hormonal abnormalities are involved in the interruption of gastric movements, hormones that delay gastric emptying time include gastrin(11)

As for the effects of gastrin on gastric emptying, when the stomach walls have been extended and some kinds of foods have come into the stomach, the secretion of a hormone named gastrin from the mucous membrane in the pyloric antrum is induced. In addition, gastrin has slight or moderate promoting effects on the motility of the stomach body and it, mostly importantly, activates the pyloric pump. However, in cases where stomach movements are abnormal, foods staying in the stomach continuously stimulate the gastric antrum to increase gastrin secretion, leading to remarkable reductions in gastric emptying rates(12).

Although dyspepsia treatment methods known thus far include drug therapy, dietetic therapy, and living habit correction(13, 14), many alternative approaches are being tried because symptoms tend to become chronic and no effective medical treatment method has been found(15).

Although the correlations between treatment methods and many symptoms of the digestive system have been fragmentally known, the correlations have not been positively proven and almost no study has been conducted in relation to the application of physical therapy to functional dyspepsia.
Therefore, this researcher conducted the present study to apply Maitland orthopedic manual physical therapy and examined the effects of the therapy on the digestive system through serum gastrin tests that can identify the motility of the stomach and functional dyspepsia symptoms.

\section{METHODS}

\section{Subjects}

The present study was conducted with 20 subjects in total, including 10 dyspepsia patients who satisfied the conditions of the modified version of the Glasgow dyspeptic questionnaire(16)(Table 1) of the dyspepsia patients who visited S Hospital in Seongnam-si between August 2014 and January 2015 and 10 healthy persons.

The general characteristics of the subjects are as follows(Table 2).

Table 1. Modified version of the glasgow dyspeptic questionnaire

1. Patients with no organic disease of which the symptoms can be explained by tests such as endoscopy

2. Patients who showed at least one symptom, such as pain centered on the epigastrium, discomfort, early satiety, epigastric satiety, sense of distension, nausea, vomiting, and belching

3. Patients with the symptoms set forth under 2. that have persisted for at least 12 weeks

Table 2 General characteristics of the subjects

\begin{tabular}{|c|c|c|c|c|}
\hline & ${ }^{\mathrm{a}} \mathrm{DG}(\mathrm{n}=10)$ & ${ }^{\mathrm{b}} \mathrm{CG}(\mathrm{n}=10)$ & Total & $p$ \\
\hline Sex(mal/female) & $2 / 8$ & $3 / 7$ & $5 / 15$ & \\
\hline Age(yr) & $20.87 \pm .64$ & $21.13 \pm .99$ & $21.00 \pm .82$ & .559 \\
\hline Hight(m) & $1.63 \pm .04$ & $1.62 \pm .04$ & $1.62 \pm .04$ & .573 \\
\hline Weight(kg) & $54.25 \pm 4.95$ & $52.75 \pm 4.20$ & $53.50 \pm 4.50$ & .524 \\
\hline $\mathrm{BMI}\left(\mathrm{kg} / \mathrm{m}^{2}\right)$ & $20.42 \pm 1.37$ & $20.15 \pm 1.25$ & $20.28 \pm 1.27$ & .684 \\
\hline
\end{tabular}

$\mathrm{M} \pm \mathrm{SD}:$ mean \pm standard deviation

${ }^{a} D G$ : dyspepsia group

${ }^{\mathrm{b}} \mathrm{CG}$ : control group 


\section{Experimental Procedure}

The subjects' agreements to their participation in the clinical study were received, their medical stories were checked, the entire study processes were informed, and the subjects were requested to stop taking drugs that might affect clinical tests at least seven days before tests.

Tests before the study were conducted on all subjects who had fasted from 22:00 on the day before the day of the tests. As tests before the study, blood was collected in the state of an empty stomach and a questionnaire survey was conducted.

Three weeks later, tests were conducted using the same method as in the tests before the study, and the results before and after the study were compared with each other.

\section{Maitland orthopedic manual physical therapy application method}

The Maitland orthopedic manual physical therapy was implemented by physical therapists that had completed the Maitland orthopedic manual physical therapy Level 2 a course. The patient was requested to take a prone position and then take the posture that felt the most comfortable to the patient. The facet joint mobilization movements used in Maitland orthopedic manual physical therapy were implemented at the level of spinal segments T6-T11 corresponding to the digestive system, and the treatment was implemented once a day for 20 minutes, five days per week for three weeks.

\section{Measurement scale}

\section{Serum gastrin measurement}

All subjects fasted at least for 10 hours before tests and approximately $5 \mathrm{~m} \ell$ of venous blood was collected from each subject on the day before and the day after the study. To reduce the errors of variations during the day, the blood was collected at 9:00 AM. The collected blood was left unattended for 30 minutes at room temperature and subjected to centrifugation for 10 minutes to separate serum, and the serum was kept at $20^{\circ} \mathrm{C}$ below zero until it was analyzed. Serum gastrin was measured by radioimmunoassay using gastrin kits from DPC(Diagnostic Products Corporation).

\section{Observation of changes in symptoms}

Questions regarding dyspepsia were composed according to the Rome II standard to indicate the intensities of seven dyspepsia symptoms, that is, epigastric pain, epigastric discomfort, early satiety, epigastric fullness, epigastric bloating, nausea and belching. The intensities were indicated with levels $0-5$ ranking scales(Table 3 ).

Table 3. Dyspepsia symptom score according to the intensity by ordinal scale

\begin{tabular}{cl}
\hline Score & \multicolumn{1}{c}{ Intensity } \\
\hline 0 & No symptom \\
1 & $\begin{array}{l}\text { Insignificant symptoms to the extent they are } \\
\text { forgotten when attention is not paid to them }\end{array}$ \\
2 & Minor symptoms appear intermittently \\
3 & Minor symptoms appear frequently \\
4 & $\begin{array}{l}\text { Symptoms persist, though they are not grave } \\
\text { to the extent they cause trouble in daily living } \\
5\end{array}$ \\
& $\begin{array}{l}\text { Symptoms are grave to the extent they cause } \\
\text { trouble in daily living }\end{array}$ \\
\hline
\end{tabular}

\section{Data analysis}

In the present study, data were analyzed using the statistical program SPSS 18.0. Frequency analyses were conducted with study subjects' general characteristics and the means and standard deviations of the characteristics were calculated. Wilcoxon matched pairs tests were conducted to examine changes between before and after the study and Mann-Whitney U Tests were conducted to identify treatment effects on the groups. To test statistical significance, the significance level was set to $a=.05$.

\section{RESULTS}

\section{Comparative analysis of the control group before and after Maitland orthopedic manual physical therapy}

In the control group, serum gastrin values significantly decreased between before and after Maitland orthopedic manual physical therapy ( $p<.01$. Among questions regarding dyspepsia, only those regarding epigastric pain and belching symptoms showed significant decreases $(\mathrm{p}<.05)$. 
The Effects of Maitland Orthopedic Manual Physical Therapy on the Digestive System

Table 4. Analyzing the effect of Maitland orthopedic manual physical therapy in CG

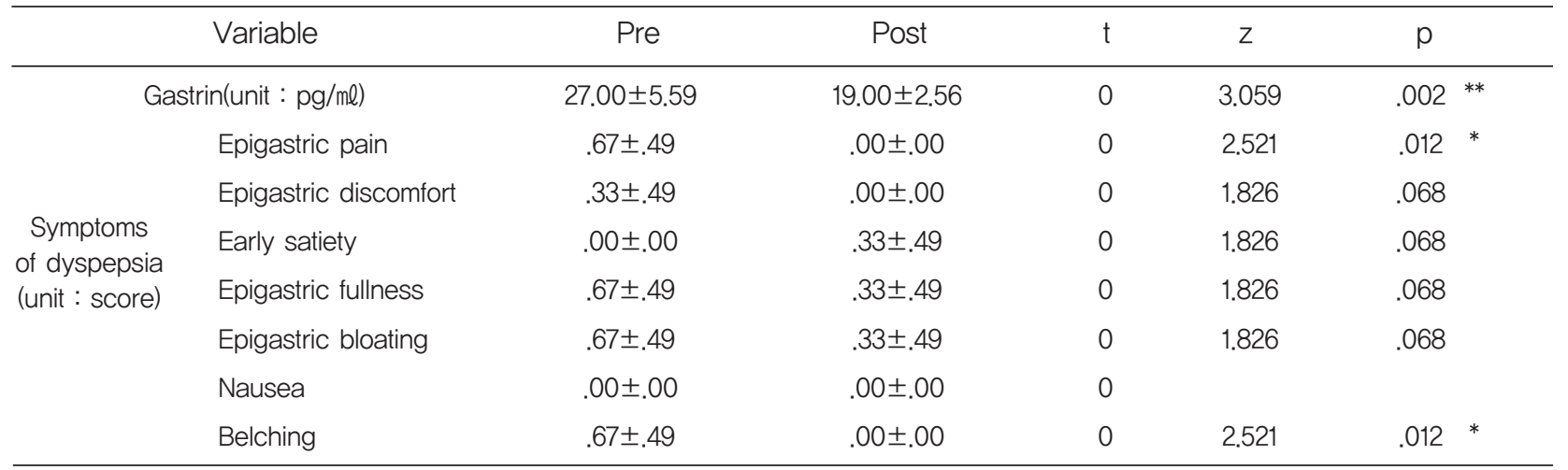

${ }^{*} p<.05,{ }^{* *} p<.01$

Comparative analysis of the dyspepsia group before and after the Maitland orthopedic manual physical therapy

In the dyspepsia group, serum gastrin values significantly decreased between before and after Maitland orthopedic manual physical therapy (p〈.01). All questions regarding dyspepsia, such as those regarding all symptoms including epigastric pain, epigastric discomfort, early satiety, epigastric fullness, epigastric fullness, nausea, and belching, showed significant decreases(p<.01).

Table 5. Analyzing the effect of Maitland orthopedic manual physical therapy in DG

\begin{tabular}{clccccc}
\hline & Variable & Pre & Post & t & z & $p$ \\
\hline & Gastrin(pg/ml) & $48.75 \pm 13.12$ & $21.00 \pm 3.77$ & 0 & 3.059 & $.002^{* *}$ \\
& Epigastric pain & $3.50 \pm .52$ & $1.00 \pm .00$ & 0 & 3.059 & $.002^{* *}$ \\
& Epigastric discomfort & $4.00 \pm .74$ & $1.75 \pm .45$ & 0 & 3.059 & $.002^{* *}$ \\
$\begin{array}{c}\text { Symptoms } \\
\text { of dyspepsia } \\
\text { (score) }\end{array}$ & Early satiety & $3.75 \pm .87$ & $1.50 \pm .52$ & 0 & 3.059 & $.002^{* *}$ \\
& Epigastric fullness & $4.00 \pm .74$ & $1.75 \pm .45$ & 0 & 3.059 & $.002^{* *}$ \\
& Epigastric bloating & $4.00 \pm .74$ & $1.00 \pm .00$ & 0 & 3.059 & $.002^{* *}$ \\
& Nausea & $2.25 \pm .87$ & $.50 \pm .52$ & 0 & 3.059 & $.002^{* *}$ \\
& Belching & $2.25 \pm .87$ & $1.25 \pm .87$ & 0 & 3.059 & $.002^{* *}$ \\
\hline
\end{tabular}

${ }^{* *} p<.01$

Analysis of differences in the effects of Maitland orthopedic manual physical therapy on the control and the functional dyspepsia groups

On reviewing these results concretely, it can be seen that the dyspepsia group indicated signifi- cant decreases in all questions regarding serum gastrin values and dyspepsia symptoms except for belching(p<.001). 
Table 6. Analyzing the differential effect of Maitland orthopedic manual physical therapy on CG and DG

\begin{tabular}{|c|c|c|c|c|c|c|}
\hline & Variable & CG(Rank Sums) & DG(Rank Sums) & $U$ & Z & $p$ \\
\hline \multirow{8}{*}{$\begin{array}{l}\text { Symptoms } \\
\text { of dyspepsia } \\
\text { (score) }\end{array}$} & Gastrin(pg/ml) & 216 & 84 & 6 & 3.811 & $.000^{* * *}$ \\
\hline & Epigastric pain & 222 & 78 & 0 & 4.157 & $.000^{* * *}$ \\
\hline & Epigastric discomfort & 216 & 84 & 6 & 3.811 & $.000^{* * *}$ \\
\hline & Early satiety & 222 & 78 & 0 & 4.157 & $.000^{* * *}$ \\
\hline & Epigastric fullness & 216 & 84 & 6 & 3.811 & $.000^{* * *}$ \\
\hline & Epigastric bloating & 222 & 78 & 0 & 4.157 & $.000^{* * *}$ \\
\hline & Nausea & 222 & 78 & 0 & 4.157 & $.000^{* * *}$ \\
\hline & Belching & 174 & 126 & 48 & 1.386 & .166 \\
\hline
\end{tabular}

${ }^{* * *} p<.001$

\section{DISCUSSION}

In the present study, to present Maitland orthopedic manual physical therapy as an intervention method for patients with dyspepsia, Maitland orthopedic manual physical therapy was implemented in patients with dyspepsia that visited the hospital. Furthermore, changes in serum gastrin and dyspepsia symptoms were observed to identify the effects of Maitland orthopedic manual physical therapy on the digestive system.

Although the relationship between delays in gastric emptying time resulting from digestive system movement disorders and digestive system symptoms are not yet clearly known, it has been known that digestive system movement disorders cause digestive systems through direct effects, such as contraction or distention, or indirect effects, such as gastroesophageal reflux, duodenogastroesophageal reflux, or gastric stagnation(17).

Hormone abnormalities are thought to be involved in gastric emptying time delays and according to information known thus far, gastrin is known as a hormone that delays gastric emptying time(18). Increases in gastrin secretion causes problems to physiological phenomena as well as gastric acid secretion involved in food digestion and gastric juice disinfection(19).

Nyren et al. measured serum gastrin on an empty stomach in a control group of healthy persons, a dyspepsia patient group, and a duodenal ulcer patient group and reported the concentration of serum gastrin was significantly higher in nonulcerating dyspepsia patients than in the control group of healthy persons and the duodenal ulcer patient group(20). Lee reported that serum gastrin values significantly decreased after Kaltenborn orthopedic manual therapy was implemented at the level of spinal segments T6-T9 that govern the stomachs of functional dyspepsia patients $(\mathrm{p}<.01)(21)$.

In the present study, serum gastrin values significantly decreased after Maitland orthopedic manual physical therapy in the control group $(p<.01)$ as well as in the dyspepsia group(p<.01).

On comparison of the results of the individual groups with each other, it could be seen that after Maitland orthopedic manual physical therapy, the serum gastrin value decreased significantly more in the dyspepsia group than in the control group $(\mathrm{p}<.01)$.

The fact the results of the present study are consistent with the results of previous studies enhanced the reliability of the study and confirmed the correlation between gastrin and dyspepsia. Gastric movement disorders due to delayed gastric emptying time and serum gastrin values are thought to be closely correlated with each other and serum gastrin is thought to play an important role in the pathological physiology of dyspepsia. Given that serum gastrin values significantly decreased after treatment based on the results of the study, the application of Maitland orthopedic manual physical therapy is thought to activate gastric movements to reduce the stimulation of excessive gastrin secretion due to stagnant foods, thereby reducing the extended gastric emptying time.

Lee reported all dyspepsia symptoms except for nausea were significantly relieved after Kaltenborn orthopedic manual therapy was implemented at the level of spinal segments T6 - T9 that 
govern the stomachs of functional dyspepsia patients(p<.05)(21).

In the control group, among questions regarding dyspepsia, only those regarding epigastric pain and belching symptoms showed significant decreases after Maitland orthopedic manual physical therapy(p<.05) and in the dyspepsia group, all questions regarding dyspepsia showed significant decreases after Maitland orthopedic manual physical therapy $(\mathrm{p}<.01)$.

On comparison of the results of individual groups with each other, it can be seen that after Maitland orthopedic manual physical therapy, the dyspepsia group indicated significantly more decreases in all questions regarding dyspepsia symptoms except for belching than the control group(p<.001).

Given the above-mentioned results, it is thought the parasympathetic system was stimulated in functional dyspepsia patients so digestive system movements were activated, leading to the relief of overall dyspepsia symptoms to the extent the patients became close to healthy persons. As these results are consistent with the results of previous studies, the reliability of the present study was enhanced.

As such, Maitland orthopedic manual physical therapy is thought to be helpful for the treatment of internal diseases without any organic cause, such as dyspepsia, by relieving symptoms, activating the motor functions of the digestive system, and shortening gastric emptying time to be close to normal time. As studies of internal disease treatment methods through physical therapeutic approaches are still insufficient in South Korea, related studies should be continued hereafter.

\section{CONCLUSION}

In the present study, to examine the effects of Maitland orthopedic manual physical therapy applied to the level of spinal segments T6-T11 corresponding to the digestive system in healthy persons and dyspepsia patients, serum gastrin values and dyspepsia symptoms were observed and the following conclusions were obtained.

Through the present study, the fact was identified that Maitland orthopedic manual physical therapy can be an effective treatment method for internal diseases, such as dyspepsia, by relieving symptoms, activating the motor functions of the digestive system, and shortening gastric emptying time to be close to normal time.

Therefore, as studies of internal disease treatment methods through physical therapeutic approaches are still insufficient in South Korea, if studies conduct various tests to investigate digestive system diseases and compare the results comprehensively, Maitland orthopedic manual physical therapy can be established as a practical internal medicinal physical therapy in clinics.

\section{REFERENCES}

1. Thompson WG, Lonstreth GF, Drossman DA, Heaton KW, Irvine EJ, Muller-Lissner SA. Functional bowel disorders and functional abdominal pain. Guts 1999; 45(2): 1143-1147.

2. Sahay P, Axon AT. Non-ulcer dyspepsia; does Helicobacter pylori matter. Postgrad J Med 1995; 71: 262-264.

3. Witteman EM, Tytgat GN. Functional dyspepsia. Neth J Med 1995; 46: 205-211.

4. Tack j, Talley NJ, Camilleri M, Holtmann G, Hu P, Malagelada JR, Stanghellini V.Functional Gastroduodenal Disorders. Gastroenterology 2006; 130(5): 1466-1479.

5. Drossman DA, Richter JE, Talley NS. The functional gastrointestinal disorder. 1st Ed. Boston, Little Brown and Company; 1994.

6. Lee SI. Functional dyspepsia. J Korean Med Assoc 2005; 48(1): 48-60.

7. Kim HO, Lee JC, Kim SK, Park JJ, Lee DH, Lee ST, Lee EH et al. Index of dyspepsia symptomsKorean IDS-K. Korean J Gastroenterol 2003; 41: 562

8. McQuid K. Dyspepsia: Pathophysioloy, Diagnosis and Management. Volume 1. 6th Ed. Philadelphia, W.B. saunder Co; 1997: 108-111.

9. Malagelada JR. Gastrointestinal motor disturbance in functional dyspepsia. Scand $J$ Gastroenterol 1991; 26: 29-34.

10. Mayer EA. New treatment of for functional pain: pharmacologic approaches. In: Syllabus of American: Gastroenterological Association Postgraduate Course. San Diego, 1995; 30: 315326.

11. MacGregor IL, Zealous DW, Martin PM. Effect of pentagastrin infusion on gastric emptying rate of solid food in man. Am J Dig Dis 1978; 23: 72. 
12. Guyton C, John EH. Text Book of Medical Physiology, 10Ed. Philadelphia, W.B. Saunders; 2002.

13. Lee JC. Treatment of Functional Dyspepsia. Journal of Neurogastroenterology and Motility 2000; 6(2): 274-279.

14. Jeong WG, Kim JH, Kim YS. Dietary Habits in Functional Dyspepsia. Korean J Family Medicine 2004; 25(1): 40-45.

15. Park SH. The Effect of Progressive Muscle Relaxation on Depression, Anxiety, and Symptoms of Patient with Functional Dyspepsia. Sung Kyun Kwan University. Master's Thesis; 2005.

16. Smith D, Gillen KM, Cochran E. Dyspepsia on withdrawal of ranitidine in Previously asymptomatic volunteers. Am J Gastoenterol 1999; 94: 1209-1213.

17. Minami H, McCallum RW. The physiology and pathophysiology of gastric emptying in humans. Gastroenterol 1984; 86: 1952.
18. Brandsborg O, Brandsborg M, Lovgreen NA, Mikkelsen K, Moller B, Rokkjaer M, Amdrup E. Influence of parietal cell vagotomy and selective gastric vogotomy on gastric emptying rate and serum gastrin concentration. Gastroenterol 1977; 72: 212 .

19. Evenepoel P. Alteration in digestion and adsorption of nutrients during profound acid supression. Best Prats Res Clin Gastroentrol 2001; 15: 234-245.

20. Nyren O, Adami HO, Bergstrom R, Gustavsson S, Loof, L, Lundavist G. Basal and food-stimulated levels of gastrin and pancreatic polypeptide in non-ulcer dyspepsia and duodenal ulcer. Scand J. Gastroenterol 1986; 21: 471.

21. Lee HJ. The Effects on the Activation of Internal Organs of Electrical Stimulation and Orthopedic Manipulative Therapy of Kaltenborn-Evjenth Concept to the T6-T9 Thoracic Vertebral Segments. Yong-in University. Master's Thesis; 2001. 